\title{
Large Scale Multiagent Simulation on the Grid
}

\author{
Ingo J. Timm \\ Center for Computing Technologies (TZI), \\ University of Bremen, Bremen, Germany \\ itimm@acm.org
}

\begin{abstract}
Simulating large scale, distributed systems of autonomous decision-makers, e.g. global logistics supply networks, is a huge challenge for hardware \& software infrastructure as well as managing the process of simulation.

Facing this challenge, we are going to introduce an architecture for large scale grid-based simulation infrastructure to enable high performance multiagent simulation experiments that deal with a huge amount of software agents, i.e. more than ten thousands of agents.
\end{abstract}

\section{Introduction}

Since the last decade, global economy and liberalization are leading to management trends as just-in-time, or reduction of vertical range of manufacture to realize shortened time-to-market and more flexible consideration of customer' demands. However, modern industrial products are often characterized by a high complexity of design, functionality and necessary manufacturing and assembly processes. This complexity may overextend the available skills, knowledge, and capacities of a single enterprise or it may force the enterprise to spend huge efforts on research and development. Thus, a current trend in logistics is, to research autonomous behaviour within logistics processes [28]. Here autonomy means the decentralised control of autonomous logistic objects in a heterarchical structure.

For analyzing, designing, managing, and controlling these networks of autonomous decisionmakers, agent technology is a promising approach. To validate this hypothesis and to explore realistic behaviour of these systems, it is necessary to model large scale application systems, which may be

\author{
Dirk Pawlaszczyk \\ Technische Universität Ilmenau, \\ Ilmenau, Germany \\ dirk.pawlaszczyk@tu-ilmenau.de
}

simulated and analyzed properly. Next to simulating the material flows of these nets it is important to integrate the information flows, as especially autonomous processes are depending on data, information, and knowledge within the environment. Current research projects within the new collaborative research center on "Autonomous Cooperating Logistic Processes - A Paradigm Shift and its Limitations" (SFB 637) at University of Bremen are studying the effects and influence of available communication networks to the interaction and performance of huge logistic networks.

In the real world, communication is costly and availability of specific communication networks, e.g. WLAN, UMTS, or GPRS, is changing while moving in the physical world. Thus, a simulation should include availability of different communication networks. This as well as the autonomous decisionmaking of physical entities (packages, trucks, etc.) requires a simulation based on multiagent systems. To analyze effects like lack of communication it is further required to simulate huge amounts of agents $\left(10^{4}\right.$ to $10^{6}$ agents). Classical simulation approaches are not capable of handling these challenges.

In this paper, we are presenting a conceptual framework and first step approach for large scale multiagent simulation based on grid computing. A toy's world example is implemented and evaluated for feasibility analysis. In section 2 and 3 basic concepts for multiagent-based simulation and grid computing will be introduced. Section 4 is summarizing requirements for large scale simulation systems as they are needed in the domain of logistics. Our approach will be introduced in section 5. The feasibility of this approach is discussed in section 6 , accompanied by a prototypical implementation of the approach. Finally, we are discussing related work in section 7 and are providing conclusions and outlook in section 8 . 


\section{Multiagent Simulation}

Simulation is an important methodology to evaluate research conclusions. Multiagent simulation can be used for simulating Multiagent systems or Multiagent systems are used for simulating distributed actors. In this paper we are focusing on simulation of distributed actors. This approach for evaluating research findings is of increasing concern especially for disciplines, where real experiments are too expensive or not feasible, i.e. simulating huge societies in social sciences. In the logistics domain, this is also a great challenge, as new algorithms try to include non trivial specialized behaviour for the individual actors. In this section we will firstly introduce multiagent systems, followed by an introduction to agent-based simulation. Finally, we will provide an overview on distributed multiagent simulation.

\subsection{Multiagent Systems}

The interest in agent technology and agent related topics has risen enormously in the last decade. Cooperative agents can act autonomously, communicate with other agents, are goal-oriented (pro-active) and are using explicit knowledge [29]. Especially in the naturally distributed production engineering domain they seem to be a promising approach.

An adequate application of co-operative agents here should meet the following three criteria [30]: natural distribution of the participating entities (e.g. resources on the shop floor), dynamic environment where structures and conditions are continually changing, and complex interaction, i.e. co-ordination between the individual entities.

Since most industrial environments meet these requirements, the use of an agent-based approach with autonomously, co-operatively and purposefully acting intelligent software units seems to be suitable to make short term and flexible reaction possible to unexpected events and disturbances within logistics.

\subsection{Agent-based Simulation}

Simulation is the imitation of a systems behaviour and structure in an experimental model to reach findings which are transferable to reality.

Basically, depending on their timely fashion, simulation approaches are discerned in continuous simulation and discrete event simulation techniques. Furthermore we can distinguish between macro and micro simulation. In contrast to macro simulation techniques like System Dynamics that is typically based on a set of mathematical equations micro simulation approaches attempt to model the individual behaviour of involved entities. Accordingly agent based simulation normally refers to time driven event simulation class where real world entities are modelled in terms of agents and system behaviour emerges from interactions between these actors. Particularly MABS is influenced by traditional simulation approaches, namely Discrete Event Simulation (DES) and Object Oriented Simulation. For a more comprehensive discussion please refer to [1].

Using an agent based world view in simulation leads to some interesting opportunities, compared to other commonly used simulation approaches, such as support of pro-active social behaviour and distributed parallel computation. Beyond this, it enables a structure-preserving intuitive modelling technique even for highly sophisticated and widely defragmented domains. Thus, MABS seems to be most convenient for simulation of tendentiously complex systems [2].

Due to these benefits MABS has been applied successfully to various problem domains like biology, logistics, health care, and production area as well as social sciences and electronic markets.

\subsection{Distributed Multiagent Simulation}

Simulation of information intensive large scale agent systems necessitates high computational power. Often it is the only feasible form of computation to distribute the simulation model, and to run agents in parallel on multiple computational nodes, to gain the required performance speed up. But, with the distribution of the simulation model to different computers new challenges arise, such as synchronizing events to ensure causality, monitoring of the distributed simulation state, load balancing, and dynamic resource allocation. So it is difficult for parallel and distributed simulation to achieve the expected performance.

However, distributed Multiagent based simulation offers new and interesting opportunities for experimenting with more complex, more realistic, and even more valuable world models. But, to exploit the expected computational power of distributed resources a suitable middleware concept must be in place first. To address the issues of failure resistance, load balancing, transparent executing of large scale simulation experiments in a domain spanning fashion grid technology seems to be most suitable [11],[12]. 


\section{Grid Computing and Peer-to-Peer}

A new approach for distributed environments commonly known as the Grid has gained broad popularity in the recent years. The Grid maintains resource sharing and interoperability among different administrative domains, by introducing a service oriented infrastructure. With its middleware the Grid offers support for a variety of applications, like high performance computation as well as parallel distributed simulation. Furthermore a vision is discussed, where the Grid evolves towards a global infrastructure to provide e-Science by introducing semantic to the Grid based on semantic web technology [8]. However, before we are going to describe our approach of a decentralized, scaleable Grid-based simulation middleware, we first want to take a closer look at the current state of affairs in Grid-Computing, and discuss possible drawbacks to our system design.

\subsection{Open Grid Service Architecture (OGSA)}

The Open Grid Service Architecture describes a set of standard protocols and interfaces to enable interoperability between different virtual organizations $^{1}$ (VO) and their proprietary grid solutions.

As a middleware approach OGSA gives support to grid services that encapsulate any resource on the grid. This is achieved by integration of Grid and Web service technology within a comprehensive framework. Upon this, standard grid functionalities for creation, registry, discovery, and lifecycle management as well as secure and reliable invocation are defined. Thus each resource is represented as a Grid Service; therefore resource discovery mainly deals with the problem of locating and querying information about useful Grid Services. The reference implementation of OGSA, the Globus Toolkit 3 (GT3), supports resource discovery by introducing a hierarchical information service [14].

Grid applications like distributed computational algorithms or parallel discrete event simulation need to be scalable and reliable to perform right. But pure hierarchical oriented resource federation approaches like GT3 can perform a bottleneck. As Grids used for complex applications, there is a strong demand for managing dynamic, large scale distributed environments.

\footnotetext{
${ }^{1}$ A virtual organization represents an institution that offers one or more Grid services.
}

Actual tests of existing Grid middleware solutions scale on very large systems not in the desired measure and turned out as error-prone [16]. Therefore appropriate technologies need to be in place concerning transport, routing and management of distributed resource networks [7]. One approach to address the concerns of scalability can be reached by decentralization.

\subsection{Peer-to-Peer Grids}

Peer-to-Peer (P2P) and the Grid basically share a common objective to exploit resources across multiple administrative domains. P2P-Systems are normally based on unstructured self organizing networks, where routing is based on forwarding. In contrast to server oriented architectures P2P-technology supports direct communication between all nodes (peers) in the network, and therefore brings connectivity to the edge of the network.

Especially in large networks with thousands of hosts efficient message routing algorithms are necessary. Overlay networks basically enable routing facilities for large networks in an ad hoc fashion, thus arise flexible and scaleable distributed applications that support reliable and fast communication channels. There are several routing-protocols in place, like Pastry, CAN, and Chord [13].

Naturally service discovery is most difficult in P2P systems. To avoid echoing, the propagation range of search requests is normally limited by a maximum number of hops in the network. Thus, interaction in $\mathrm{P} 2 \mathrm{P}$-systems tends to be localized. To overcome this limitation we could take the best of both worlds (Grid and $\mathrm{P} 2 \mathrm{P}$ ) to implement non-hierarchical decentralized grid systems, commonly known as P2P-Grids [9], [10]. P2P-Grid promises to be an especially potent mechanism for scaling up distributed simulation.

In 2001, Sun Microsystems started the JXTA project, an open source standardization initiative that is intented to offer a common P2P infrastructure [15]. It mainly consists of a stack of open, XML-based protocols to support interaction between peers. Core capabilities like searching, indexing, grouping and security are part of the JXTA core. JXTA is independent from the underlying transport protocols, i.e. it can be build up on top of a normal TCP/IP as well as Bluetooth connection. Peers are organized within peer groups. Service discovery is supported via rendezvous peers, which provide information on peers they are aware of on the network.

To summarize, JXTA addresses some important issues regarding grid scalability and decentralization. 
Furthermore with peer groups a suitable metaphor for the virtual organizations is in place [14]. Thus, an implementation of JXTA within OGSA seems to be a promising approach for a service oriented infrastructure, that enables higher level services and applications such a Multiagent based simulation.

\section{Requirements}

The goal of MABS on the grid is to take advantage of grid technology to speed up simulation experiments. In the following, we are presenting requirements, which have been identified for simulation approaches in the logistic domain. In the collaborative research center on autonomous cooperating logistic processes, there are 45 researchers of four faculties working in 12 interdisciplinary projects. Simulation systems within this context should range from simulating communication computationally restricted physical entities (e.g. RFID-tags) to autonomous, deliberative decision makers like agents. The requirements for a large scale simulation system have been identified by analysis of existing systems, identification of necessary concepts for simulation control and simulation models. Additionally, each project returned a well-structured questionnaire ${ }^{2}$, which should provide information on project-specific as well as applicationspecific requirements.

The main requirement is that MABS has to be capable of simulating large numbers of autonomous entities (up to $10^{6}$ ) for simulating realistic scenarios. This leads to further requirements, e.g. semantic load balancing. For optimal performance, semantic relations between tasks should be identified and used for load balancing. In the logistics domain, there should be load balancing with respect to geographical distribution. Next to these requirements, MABS should be domain independent, such that the simulation control and management could be used in different applications.

A requirement to a grid based simulation infrastructure is to support a wide range of agent based simulation experiments. Furthermore seamless transition of simulations models to real world applications should be feasible. The simulation infrastructure should allow for distributed simulation

\footnotetext{
${ }^{2}$ The questionnaire has been returned by 10 projects within the Collaborative Research Center on "Autonomous Logistic Processes - A Paradigm Shift and its Limitations" (DFG-SFB 637) in 2004.
}

models and should enable high scalable simulation. The scalability should be dynamic during runtime, i.e. the system should allocate new resources whenever necessary and free resources whenever possible.

\section{MABS on the Grid}

Grid based computing is introducing an innovative infrastructure for large scale applications. In the context of simulation, it seems to be promising to use the grid paradigm for creating an infrastructure and the MABS approach for simulation. In combination they are building a large scale simulation infrastructure. In the next paragraphs, we are introducing an architecture for grid based MABS, with special focus on service discovery and linking as well as the simulation process.

\subsection{Architecture}

The system design represented next is intended to provide scalability, transparency, modularity and flexibility. Therefore, we propose a service-oriented, decentralized, grid-based approach to maintain distributed simulation experiments.

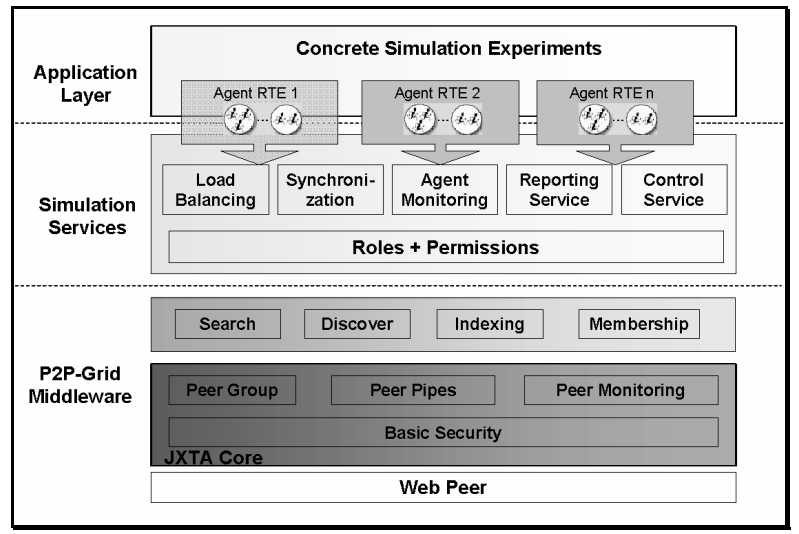

Figure 1. Layered Architecture

Within the current system architecture (Figure 1) various layers are recognized, each meant to fulfil a defined task. The P2P-Grid layer provides functionalities to support the required resource infrastructure. It is composed of services that enable discovery, linking and management of computational resources on the Grid in a transparent way. Most of these services are already supported within JXTA framework. To be "Grid-aware", i.e. to be compliant with OGSA grid service specification, some services such as the discovery service needs to be extended. 

distributed simulation are introduced. However, the presented work is a first step approach yet. Nevertheless, a first prototype for feasibility issues has been implemented.

\subsection{Feasibility Study}

In a first attempt, feasibility of the proposed design has been analysed using the well known travelling salesman problem, whereby computation is based on an evolutionary algorithm (Figure 4). To test the scalability of the distributed service-oriented approach we executed several thousands computation runs while changing the number of nodes in the JXTA network. Accordingly, results are crucially determined by the parallelism of the application that is caused by the distribution overhead. Nevertheless, we could achieve a nearly linear speed, decreasing computation time by 30 percent in average when doubling hosts(Figure 5).

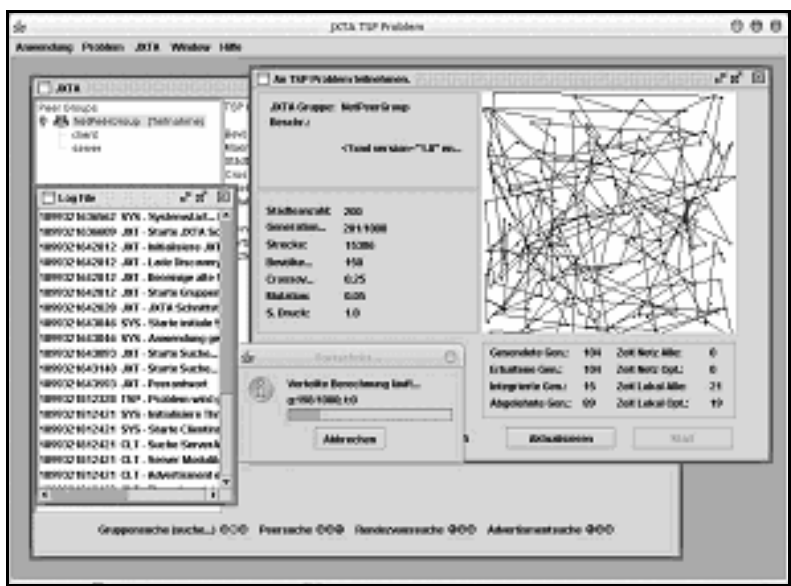

Figure 4. User-interface of P2P-Grid-Prototype

\subsection{Setting up a distributed simulation}

As pointed out, the simulation model needs to be transferred to the according agent runtime environments hosted by different peers of our P2PGrid. We aimed at investigating the behaviour of the distributed simulation system under increasing agent loads. Therefore we studied the growth of the network load as a function of the number of agents in a P2PGrid (Figure 6) ${ }^{3}$ within a second preliminary test series. Agents have been transferred either using stream objects or alternatively by using an xml-based transmission format. As experimental results show, the overall payload during initialization stage of our

${ }^{3}$ Our test environment comprises on a cluster of 5 machines connected by a 100 Mbit Ethernet switch, each hosting a JADE-based agent runtime environment. simulation process doesn't really affect the peernetwork. Furthermore, there is no significant difference with respect to the resulting payload of transfer formats. Both techniques were stressing the network similarly.

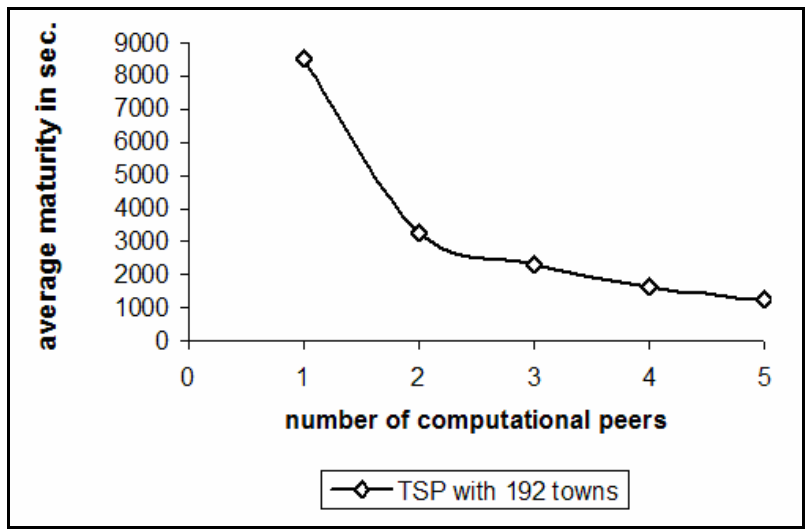

Figure 5. Distributed TSP solved in a P2P-Grid

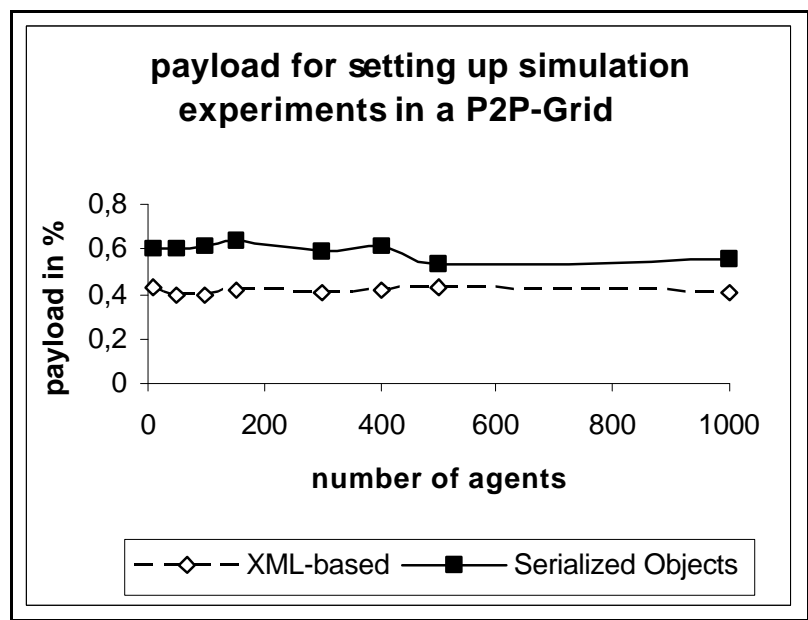

Figure 6. Payload during initialization stage

The prototypical implementation as well as the simulation results corroborate the thesis adequate for MABS with respect to discovery of computational host, distribution, data mining, and communication. We are confident to successfully transfer the findings from our first studies to simulation of large scale agent-based models, with several thousand agents, running on different JXTA-based peers inside of hosted agent runtime environments.

\section{Related Work}

There are several contributions in place related to multiagent simulation - too much to treat them all in detail here. Briefly, most existing toolkits attempt to 
exploit particular aspects of some application domain, like ecological systems, logistics, manufacturing or social sciences. A short survey of main themes and simulation toolkits is given in [26]. Moreover existing MAS-based simulation frameworks are restricted to a single computer and normally do not permit support to distributed simulation.

A plenty of current contributions to distributed simulation of agent models tend to be rash and barely projected and therefore has yielded relatively low performance [17]. Often these approaches use a simple time driven approach, and therefore do not fully utilize intrinsic parallelism of multi agent technology. Explicit consideration of discrete events was introduced within some specialized agent toolkits. In JAMES for example a centralized event oriented simulation model is used [25]. Beyond this some conventional agent-simulators has been extended to support distributed parallel execution like Distributed GenSim [27] and MACE-3J[18]. Latter one uses proxies for interaction of hierarchically ordered simulation nodes. Another approach utilizes CORBA as a middleware to agents based simulation experiments [24]. In [20] a middleware for parallel agent discrete event simulation (MPADES) is introduced, that facilitates a conservative simulation approach, to ensure event causality, with a centralized environment model.

Other approaches propose to connect AgentSimulators by using the High Level Architecture (HLA), a framework that offers interoperability among different simulation environments [19], [23]. With HLA simulators, referred to as federates, can be integrated into a global simulation context known as federation. A strict hierarchical tree-oriented model is used to structure respective federates. Communication between certain simulators is enabled be predefined gateways. Thus HLA can be considered as a centralized coordination approach to distributed simulation resources.

Finally there are some approaches which collaborate in integration of none agent based discrete event simulation within a grid infrastructure [22], [6]. Currently there is only one contribution that explicitly deals with MAS simulation on the grid [18]. Within MACE3J services are deployed with GT3 to perform progressive scaling of simulation, using a hierarchical resource federation approach. But this work is still in progress.

To summarize, up to now there are only a few projects that deal with Distributed Multiagent Simulation in common, and even fewer are related to
Agent-based simulation on the Grid. Furthermore current approaches of a simulation middleware normally tend to be almost centralized, with simulators organized in a hierarchical fashion. Moreover numerous contributions only offer limited support to smooth synchronization techniques as well as pure load balancing facilities. Thus they are unlikely to scale well to a large number of agents. To date none covers the requirements for scaleable grid enabled multi purpose simulation environment to Multi Agent Systems.

\section{Conclusion and Future Work}

In this paper, we introduced an approach for large scale simulation based on the integration of grid computing and MABS. Grid based computing is introducing an innovative infrastructure for large scale applications. However, MABS is providing a suitable approach for simulation of societies or systems consisting of autonomous decision-makers. This approach enables the user to benefit from the easy modelling approach of MABS and the high scalability of grid computing. In a first prototype, we proofed feasibility of the abstract architecture introduced in this paper. Nevertheless, simulation of realistic scenarios is missing for evaluating the quality of service as well as performance of grid based MABS.

In the Collaborative Research Center we are developing a large scale simulation system, which will be based on experiences gained with this prototypical implementation and will implement the architecture presented in this paper. Furthermore, it will include semantic load balancing on the basis of geographical regions. Next to the implementation it is planned to refine the architecture and specify services needed for simulation.

\section{Acknowledgement}

Parts of this research is funded by the German Research Foundation (DFG) within the Collaborative Research Centre 637 "Autonomous Cooperating Logistic Processes: A Paradigm Shift and its Limitations" (SFB 637). Additional information can be found at http://www.sfb637.uni-bremen.de/.

\section{References}

[1] P. Davidsson, Multi Agent Based Simulation: "Beyond Social Simulation", MABS 2000, LNAI 1997, S. Moss and 
P. Davidsson (Eds.), Springer-Verlag Berlin Heidelberg, 2000, pp. 97-107.

[2] S. Moss, "Messy Systems - The Target for Multi Agent Based Simulation", MABS 2000, LNAI 1979, S. Moss and P. Davidsson (Eds.), Springer-Verlag Berlin Heidelberg, 2000, pp. 1-14.

[3] K. Popov, et al, "Parallel Agent-Based Simulation on a Cluster of Workstation", Euro-Par 2003, H. Kosch, H. Böszörményi, H. Hellwagner (Eds.), Springer-Verlag Berlin Heidelberg, 2003, pp. 470-480.

[4] R. M. Fujimoto, Parallel and Distributed Simulation Systems, John Wiley \& Sons Inc., 2000.

[5] F. Kluegl, Multi Agent Simulation - Concepts, Tools, Application (in german), Addison Wesley, Muenchen, 2001.

[6] C. Zhang, Y. Liu et al, "Integration of Distributed Simulation into the OGSA Model", M. Li et al, (Eds.) GCC 2003, LNCS 3032, Springer-Verlag Berlin Heidelberg, 2004, pp. 200-204.

[7] I. Foster, Adriana Iamnitchi, "On Death, Taxes, and the Convergence of Peer-to-Peer and Grid-Computing", http://people.cs.uchicao.edu/ anda/papers/foster_grid_vs_p2 p.pdf, 2003.

[8] D. De Roure, M. A. Baker, N. R. Jennings, and N. R. Shadbolt, "The Evolution of the Grid", in Berman, F. Fox, G. and Hey, AJG, Eds. Grid Computing - Making the Global Infrastructure a Reality, Jon Wiley and Sons Ltd., 2003, pp. 65-100.

[9] G. Fox, S. Pallickara, and X. Rao, "Towards Enabling Peer to Peer Grids", Journal of Concurrency and Computation: Practice and Experience. ACM JavaGrande ISCOPE Special Issue, 2004.

[10] D. Talia, P. Trunfio, "Toward a Synergy between P2P and Grids", Issue of IEEE Internet Computing. http://dsonline.commputer.org/0307/d/wp4p2p.htm, 2003.

[11] C. Kim, T. Lee et al, Grid-Based Parallel and Distributed Simulation Environment, V. Malyshkin (Ed.): PaCT 2003, LNCS 2763, Springer-Verlag Berlin Heidelberg, 200 pp. 503-508.

[12] C. Zhang, Y. Liu et al, "Integration of the Distributed Simulation into the OGSA Model", M. Li et al. (Eds.): GCC 2003, LNCS 3032, Springer-Verlag Berlin Heidelberg, 2004, pp. 200-204.

[13] M. Kelaskar, V. Matossian, et al, "A Study of Discovery Mechanisms for Peer-to-Peer Applications", Proceedings of the $2^{\text {nd }}$ IEEE/ACM International Symposium on Cluster Computing and the Grid, Berlin, Germany, IEEE Computer Society Press, May 2002, pp. 444-445.

[14] I. Foster, C. Kesselmann et al, "Physiology of the Grid". http://www.globus.org/research/papers/ogsa.pdf, June 2002.

[15] B. J. Wilson, JXTA, New Riders Publishing, 2002.

[16] A. Reinefeld, F. Schintke, "Grid Services - Web Services zur Nutzung verteilter Ressourcen", (in german) Informatik Spektrum Band 27, SpringerVerlag, April 2004, pp. 129-135. [17] M. Lees, B. Logan et al, "Distributed Simulation of MAS", Proceedings of the Joint Workshopon Multi-
Agent \& Multi-Agent-Based Simulation, Autonomous Agents \& Multi Agent Systems (AAMAS) New York, USA, July 2004, pp. 21-30.

[18] L. Gasser, K. Kakugawa et al, "Smooth Scaling Ahead: Progressive MAS Simulation from Single PCs to Grids", Proceedings of the Joint Workshop on Multi-Agent \& MultiAgent-Based Simulation, Autonomous Agents \& Multi Agent Systems (AAMAS) New York, USA, July 2004, pp. $1-10$.

[19] E. Göktürk, F.Polat, "Implementing Agent Communication for a Multi-agent Simulation Infrastructure on HLA", A. Yazici, and C. Sener (Eds.): ISCIS 2003, LNCS 2869, Springer-Verlag Berlin Heidelberg, 2003, pp. 619-626.

[20] P. Riley, "MPADES: Middleware for Parallel Agent Discrete Event Simulation", G.A. Kaminka, P.U. Lima, and R. Rojas (Eds.): RoboCup 2002, LNAI 2752, Spring-Verlag Berlin Heidelberg, 2003, pp. 162-178.

[21] R.H. Bordini, F.Y. Okuyama et al, "The MAS-SOC Approach to Multi-agent Based Simulation", G. Lindemann et al. (Eds.): RASTA 2002, LNAI 2934, Springer-Verlag Berlin Heidelberg, 2004, pp. 70-91.

[22] C.-H. Kim, and T.-D. Long et al, "Grid-Based Parallel and Distributed Simulation Environment", V. Malyshkin (Ed.): PaCT 2003, LNCS 2763, Springer-Verlag Berlin Heidelberg, 2003, pp.503-508.

[23] M. Lees, B. Logan, "Simulating Agent-Based Systems with HLA: The Case of SIM_AGENT - Part II", www.cs.nott.ac.uk/ mhl/papers/Lees++:03a.pdf, 2003.

[24] K.-H. Kim, W.-S. Kang, "CORBA-Based, Multithreaded Distributed Simulation of Hierarchical DEVS Models: Transforming Model Structure into a Nonhierarchical One", A. Laganà et al. (Eds.): ICCSA 2004, Springer-Verlag Belin Heidelberg, 2004, pp. 167-176.

[25] A.M. Uhrmacher, B. Schattenberg, "AGENTS IN DISCRETE EVENT SIMULATION", A. Bargiela and E. Kerckhoffs (Eds.) Proceedings of the 10TH European Simulation Symposium "Simulation in Industry - Simulation Technology: Science and Art”' (ESS'98), SCS Publications, Ghent, 1998, pp. 129-136.

[26] L. Gasser, K. Kakugawa, "MACE3: Fast Flexible Dirstributed Simulation of Large-Grain Multi-Agent Systems“", AAMAS'02, Bolonga, Italy, July 2002.

[27] J. Anderson, "A Generic Distributed Simulation System for Intelligent Agent Design and Evaluation”, Proceedings of the AI, Simulation \& Planning In High Autonomy Systems, Arizona 2000.

[28] B. Scholz-Reiter, K. Windt, M. Freitag: Autonomous logistic processes: New demands and first approaches. In: Monostori, L. (ed.): Proceedings of the 37th CIRP International Seminar on Manufacturing Systems. Budapest, Hungaria, 2004, pp. 357-362.

[29] G. Weiss, "Multiagent Systems - A Modern Approach to Distributed Artificial Intelligence", The MIT Press: Cambridge, Massachusetts, 1999.

[30] H.-J. Mueller, „Towards Agent Systems Engineering”, Data \& Knowledge Engineering, 23(3), 1997, pp. 217-245. 